\title{
Pharmacokinetic Modelling to Predict FVIII:C Response to Desmopressin and Its Reproducibility in Nonsevere Haemophilia A Patients
}

\author{
Lisette M. Schütte $^{1}$ Reinier M. van Hest ${ }^{2}$ Sara C. M. Stoof ${ }^{1}$ Frank W. G. Leebeek ${ }^{1}$ \\ Marjon H. Cnossen ${ }^{3}$ Marieke J. H. A. Kruip ${ }^{1}$ Ron A. A. Mathôt ${ }^{2}$
}

${ }^{1}$ Department of Haematology, Erasmus University Medical Centre,

Address for correspondence R. A. A. Mathôt, PharmD, PhD, Academic Rotterdam, The Netherlands Medical Centre, P.O. 22660, 1100 DD Amsterdam, The Netherlands

2 Department of Hospital Pharmacy, Academic Medical Centre, (e-mail: r.mathot@amc.nl).

Amsterdam, The Netherlands

${ }^{3}$ Department of Paediatric Haematology, Sophia Children's Hospital,

Erasmus University Medical Centre, Rotterdam, The Netherlands

Thromb Haemost 2018;118:621-629.

\begin{abstract}
Keywords

- desmopressin

- factor VIII

- haemophilia A

- haemostasis

- pharmacokinetics
\end{abstract}

Background Nonsevere haemophilia A (HA) patients can be treated with desmopressin. Response of factor VIII activity (FVIII:C) differs between patients and is difficult to predict.

Objectives Our aims were to describe FVIII:C response after desmopressin and its reproducibility by population pharmacokinetic (PK) modelling.

Patients and Methods Retrospective data of 128 nonsevere HA patients (age 7-75 years) receiving an intravenous or intranasal dose of desmopressin were used. PK modelling of FVIII:C was performed by nonlinear mixed effect modelling. Reproducibility of FVIII:C response was defined as less than $25 \%$ difference in peak FVIII:C between administrations.

Results A total of $623 \mathrm{FVIII:C} \mathrm{measurements} \mathrm{from} 142$ desmopressin administrations were available; 14 patients had received two administrations at different occasions. The FVIII:C time profile was best described by a two-compartment model with firstorder absorption and elimination. Interindividual variability of the estimated baseline FVIII:C, central volume of distribution and clearance were 37,43 and $50 \%$, respectively. The most recently measured FVIII:C (FVIII-recent) was significantly associated with FVIII:C response to desmopressin $(p<0.001)$. Desmopressin administration resulted in an absolute $\mathrm{FVIII:C}$ increase of $0.47 \mathrm{IU} / \mathrm{mL}$ (median, interquartile range: $0.32-0.65$ $\mathrm{IU} / \mathrm{mL}, n=142$ ). FVIII:C response was reproducible in 6 out of 14 patients receiving two desmopressin administrations.

Conclusion FVIII:C response to desmopressin in nonsevere HA patients was adequately described by a population PK model. Large variability in FVIII:C response was observed, which could only partially be explained by FVIII-recent. FVIII:C response was not reproducible in a small subset of patients. Therefore, monitoring FVIII:C around surgeries or bleeding might be considered. Research is needed to study this further. received

June 6, 2017

accepted after revision

December 1, 2017
Copyright () 2018 Schattauer
DOI https://doi.org/ 10.1160/TH17-06-0390. ISSN 0340-6245. 


\section{Introduction}

Haemophilia A (HA) is a hereditary bleeding disorder, characterized by a deficiency of coagulation factor VIII (FVIII). Nonsevere HA patients (FVIII $\geq 0.01-0.40 \mathrm{IU} / \mathrm{mL}$ ) suffer from a bleeding risk after minor trauma and during surgical interventions. To prevent or treat bleeding, FVIII concentrate or desmopressin can be administered. Desmopressin is a synthetic analogue of vasopressin and improves haemostasis by release of endogenous FVIII and von Willebrand factor (VWF) from storage sites in the endothelium. ${ }^{1,2}$ Desmopressin is inexpensive, widely available and only sporadically causes adverse reactions. $^{3}$

In general, a desmopressin test dose is administered to nonsevere HA patients to evaluate individual FVIII:C response. This is important as the absolute increase of FVIII:C following desmopressin administration varies between patients. Moreover, not every patient reaches adequate FVIII:C levels to prevent or treat bleeding. Several factors have been identified that seem to influence the FVIII:C response. The lowest FVIII:C level ever measured (FVIII-lowest) has a positive correlation with the absolute increase in FVIII:C after desmopressin. ${ }^{4-6}$ In addition, multiple F8-gene mutations have been reported to affect response. $^{7,8}$ However, reliable prediction of individual FVIII:C response remains difficult. In addition, reproducibility is an issue. Reproducibility was previously defined as follows: a maximum deviation of $20 \%$ of a single FVIII:C response from the average FVIII:C response in one patient. Rodeghiero et al found this to be the case in $70 \%$ of patients. However, these observations were made in only 10 adult HA patients. $^{9}$

Population pharmacokinetics (PK) may be a valuable new approach to quantify and predict the FVIII:C response to desmopressin. Previous studies have shown the merit of developing population PK models for individualizing prophylactic and perioperative dosing of FVIII concentrate treatment in severe and moderate-severe HA patients. ${ }^{10-12}$ A large advantage of population PK modelling is that only a limited number of samples per patient is required as all patient data are analysed simultaneously. In addition, patients with a variable number of samples available can be included in the analysis. With population PK modelling, the interindividual variability (IIV) in FVIII:C response can be quantified. In addition, it may lead to the identification of covariates which explain this IIV in the observed response, enabling prediction of desmopressin response in individual patients.

Our aim was to assess the population PK of FVIII:C following administration of desmopressin, to quantify the IIV in FVIII:C response and to identify covariates explaining this IIV. In addition, reproducibility of FVIII:C response was assessed.

\section{Patients and Methods}

This study was a retrospective cohort study and was not subjected to the Medical Research Involving Human Subjects Act (WMO).

\section{Patient Inclusion}

All nonsevere HA patients (FVIII $\geq 0.01 \mathrm{IU} / \mathrm{mL}$ ) in the Erasmus University Medical Centre, Rotterdam were screened. Patients were included if they had received at least one administration of desmopressin with at least one FVIII:C measurement around the desmopressin administration. Patients were only included if they had had at least 3 days without desmopressin or FVIII concentrate treatment prior to desmopressin administration.

\section{Desmopressin Administration}

Patients either received a standard dose of $0.3 \mu \mathrm{g} / \mathrm{kg}$ desmopressin intravenously without a maximum or capped dose, which was administered in 30 minutes or an intranasal dose of desmopressin in a standard dose of $300 \mu \mathrm{g}$. FVIII:C was measured by a one-stage assay. In case of a desmopressin test dose, FVIII:C was measured prior to desmopressin administration and at multiple time points after administration. The time points for blood sampling were $0.5,1,2,3,4,6$ and 24 hours after desmopressin administration. The number of obtained samples differed per patient depending on the protocol, with a maximum of seven samples after desmopressin administration. In patients receiving desmopressin as treatment for bleeding or as prophylaxis before surgical interventions, FVIII:C was measured before and between 0.5 and 2 hours after administration.

\section{Data Collection}

All data were extracted from patient files. Demographic data including height and body weight were documented. The following FVIII:C measurements were collected: FVIII-lowest, the lowest FVIII:C ever measured in a patient (before or after desmopressin administration), and the most recently measured FVIII:C (FVIII-recent). FVIII-recent was defined as the most recent FVIII: $C$ measurement, obtained $\geq 1$ day before the desmopressin administration and in the absence of a treatment effect on FVIII values before the desmopressin administration ( - Fig. 1a). In addition, the reason for desmopressin administration was documented (test, treatment or prophylaxis). When available, the following data were collected: VWF antigen (VWF:Ag), both historical and after desmopressin administration, blood type, F8-gene mutation, platelet count and activated partial thromboplastin time (aPTT).

\section{Population Pharmacokinetic Modelling}

A compartmental population PK model was developed describing the time profile of FVIII:C after desmopressin administration, including its IIV and intraindividual variability using the software package NONMEM (7.1.2; Icon Development Solutions, Ellicott City, Maryland, United States). Since no actual FVIII concentrate was administered, the dose was arbitrarily set to unity. Consequently, the estimated PK parameters represent apparent PK parameters. The first step in model development was to test how many compartments best fitted the data. For this purpose, all data were simultaneously fitted, first to a one-compartment model and subsequently to a two-compartment model and 

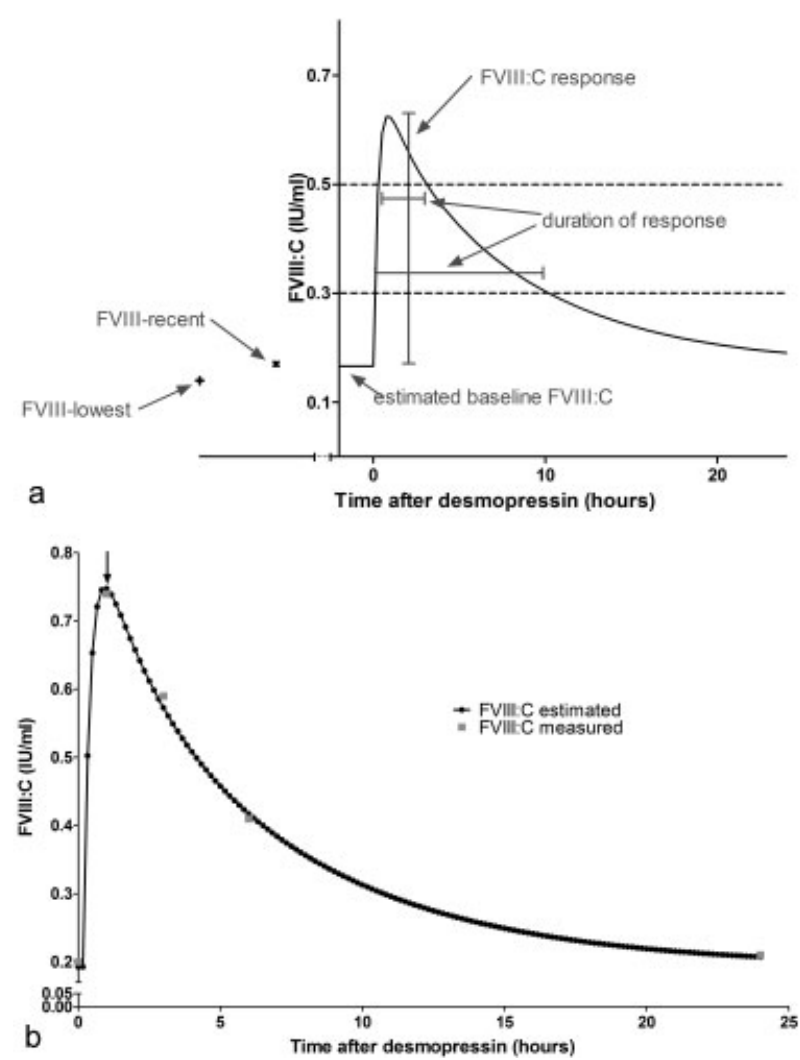

Fig. 1 (a) Definitions of FVIII parameters depicted in a curve of FVIII: $\mathrm{C}$ response to desmopressin. FVIII, factor VIII; FVIII:C, response of factor VIII activity; FVIII-lowest, lowest FVIII:C ever measured; FVIIIrecent, most recently measured FVIII:C $\geq 1$ day before desmopressin administration without previous treatment; baseline FVIII, estimated FVIII:C directly before desmopressin administration; FVIII response, estimated peak FVIII:C minus estimated baseline FVIII; duration of response, time FVIII:C is above 0.5 or $0.3 \mathrm{IU} / \mathrm{mL}$ (in hours). (b)

Example of an individual response-time curve. Highest point in the curve is the estimated peak FVIII:C (arrow). The individual pharmacokinetic estimates of this patient: estimated baseline FVIII:C $=0.19$ $(\mathrm{IU} / \mathrm{mL})$; volume of distribution $=1.49(\mathrm{~L})$; clearance $=0.27(\mathrm{~L} / \mathrm{h})$.

the goodness-of-fit between both was compared, as described later. Estimated parameters were a first-order absorption rate constant $\left(\mathrm{k}_{\mathrm{a}}\right)$, clearance $(\mathrm{CL})$ and central volume of distribution (V1). In case of a two-compartment model, peripheral volume of distribution (V2) and intercompartmental clearance $(\mathrm{Q})$ were also estimated. As nonsevere HA patients were included, patients had a measurable FVIII:C before administration of desmopressin. Therefore, estimation of baseline FVIII:C was performed in the population analysis (-Fig. 1a). In the model, FVIII:C increase following desmopressin administration was added to the estimated baseline FVIII:C. More details on the modelling methodology are provided in - Supplementary Table 1.

Whether IIV and intraindividual variability could be explained by patient and/or haemophilia characteristics were tested in a covariate analysis (see - Supplementary Table 1, Eqs. 4 and 5 for further details). Correlations were first tested in a univariate analysis for the following covariates: FVIII-lowest, FVIII-recent, F8-gene mutation domain, historical VWF:Ag, VWF:Ag during desmopressin administration, aPTT, platelet count, blood type, body weight, age, route of administration and indication for desmopressin administration (test, bleeding, prophylaxis before surgery). Subsequently, a multivariate analysis was performed with all statistically significant covariates from the univariate analysis with forward addition and backward elimination to obtain the final population model.

Improvement of the population model by adding a compartment or by implementing an association between a covariate and a PK parameter was evaluated on the basis of the likelihood ratio test. A $p$-value $<0.001$ was considered statistically significant, except for the univariate covariate analysis, where $p<0.05$ was considered statistically significant. Furthermore, goodness-of-fit was judged by visual inspection of diagnostic plots as is the golden standard in population PK analysis. ${ }^{13}$ These diagnostic plots were created using Pirana (version 2.9.0) and Xpose (version 4.3.2, Nicolas Jonsson and Mats Karlsson, Uppsala, Sweden). ${ }^{13-15}$ A visual predictive check (VPC) was done to investigate whether the model adequately describes the observed time course of FVIII:C response and the variability within it. VPC analyses were performed using Perl speaks NONMEM (PsN, version 3.5.3, Uppsala, Sweden). ${ }^{16}$

\section{FVIII:C Response}

The FVIII: $\mathrm{C}$ response to desmopressin was defined as absolute increase of FVIII:C (expressed as $\mathrm{IU} / \mathrm{mL}$ ) from the estimated baseline FVIII:C, that is, the difference between peak FVIII:C after desmopressin and baseline FVIII:C. To calculate the peak FVIII:C, individual estimates of the PK parameters (baseline FVIII:C, CL and V1) were obtained by Bayesian analysis using the final population PK model. Subsequently, these individually estimated PK parameters were used to construct the individual FVIII:C response-time curve by calculating the individual FVIII:C response every 10 minutes from the moment of desmopressin administration until 24 hours thereafter. The highest estimated FVIII:C response was taken as the peak FVIII:C (-Fig. 1b). Duration of response was calculated in patients with peak FVIII:C above certain target FVIII:C levels. These target levels were chosen according to the guidelines for surgeries and other procedures. ${ }^{17}$ Partial response was defined as the time period of FVIII:C above $0.30 \mathrm{IU} / \mathrm{mL}$ and complete response was defined as the time period above $0.50 \mathrm{IU} / \mathrm{mL}$ ( - Fig. 1a). The moment from reaching the target levels until the individually estimated FVIII:C response dropped below these target levels was taken as the duration of response. Reproducibility was defined as a difference in estimated peak FVIII:C of less than $25 \%$ between separate desmopressin administrations. This cutoff value is common in bioequivalence studies. ${ }^{18}$

\section{Results}

In total, 128 nonsevere HA patients were included. Median age was 28 years (range: $7-75)$ with $34(24 \%)<18$ years at the time of desmopressin administration $(N=142)$. Median FVIII-lowest was $0.10 \mathrm{IU} / \mathrm{mL}$ (interquartile range [IQR]: 0.04$0.18 \mathrm{IU} / \mathrm{mL}$ ). Twenty-one patients had moderate HA (16\%) 
and 107 mild HA (84\%). In 126 administrations (92\%), desmopressin was given intravenously and in 11 administrations (8\%) intranasally. In 14 patients, repeated desmopressin administrations were performed with available FVIII: $\mathrm{C}$ measurements. The specific reasons for repeated monitoring were unknown in 7 out of 14 patients. In three patients, only limited FVIII:C measurements were available after the first desmopressin administration. In these patients, a more extensive test was performed at a later time point to evaluate long-term response. In four patients, one of the administrations was performed intranasal and the other one intravenously, and therefore, response was re-evaluated. In total, 142 desmopressin administrations were included, 83\% $(n=109)$ of which was a test administration, $7 \%(n=9)$ prophylactic and $10 \% \quad(n=13)$ treatment for bleeding. - Table 1 shows all demographic data. Together all desmopressin administrations resulted in 623 FVIII:C measurements (-Fig. 2). - Supplementary Tables 1 and 2 summarize the number of FVIII:C measurements collected per sample time and per patient.

Table 1 Demographic data

\begin{tabular}{|c|c|}
\hline Patient characteristics & \\
\hline Age (y) (range) $(n=142)$ & $28(7-75)$ \\
\hline Weight $(\mathrm{kg})$ (range) $(n=111)$ & $75(26-120)$ \\
\hline \multicolumn{2}{|l|}{ Mutation domain $(n=110)$} \\
\hline A1 & $24(22 \%)$ \\
\hline $\mathrm{A} 2$ & 37 (34\%) \\
\hline A3 & $5(5 \%)$ \\
\hline B & $7(6 \%)$ \\
\hline C1 & $26(24 \%)$ \\
\hline $\mathrm{C} 2$ & $11(10 \%)$ \\
\hline Moderate HA $(n=128)$ & $21(16 \%)$ \\
\hline Blood type O $(n=113)$ & $48(42 \%)$ \\
\hline FVIII-lowest $(\mathrm{IU} / \mathrm{mL})(\mathrm{IQR})(n=128)^{\mathrm{a}}$ & $0.10(0.04-0.18)$ \\
\hline FVIII-recent (IU/mL) (IQR) $(n=120)^{\mathrm{a}}$ & $0.15(0.08-0.24)$ \\
\hline $\begin{array}{l}\text { Historical VWF:Ag (IU/mL) (IQR) } \\
(n=104)\end{array}$ & $1.13(0.91-1.55)$ \\
\hline Intranasal administration $(N=137)$ & $11(8 \%)$ \\
\hline \multicolumn{2}{|l|}{$\begin{array}{l}\text { Indication for desmopressin } \\
\text { administration }(n=131)^{\mathrm{b}}\end{array}$} \\
\hline Test dose & $109(83 \%)$ \\
\hline Prophylaxis & $9(7 \%)$ \\
\hline Treatment for bleed & $13(10 \%)$ \\
\hline
\end{tabular}

Abbreviations: IQR, interquartile range; HA, haemophilia A; FVIII-lowest, lowest ever measured FVIII value; FVIII-recent, most recently FVIII value, $\geq 1$ day before desmopressin administration; VWF:Ag, von Willebrand factor antigen.

Note: Data were expressed as median with (IQR) or as frequency (\%).

${ }^{a}$ In 15 patients, FVIII-lowest was equal to FVIII-recent.

${ }^{b}$ All desmopressin administrations. Fourteen patients had two administrations included in this study, total is 142 .

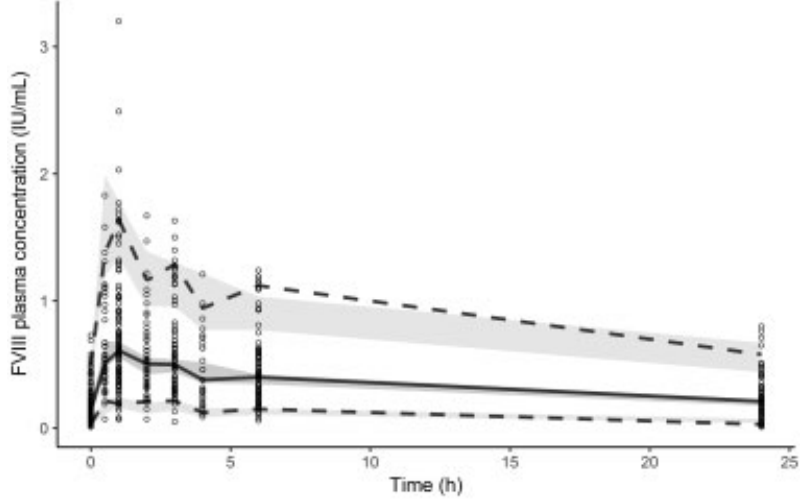

Fig. 2 VPC: the 5th, 50th and 95th percentiles of FVIII:C responses as measured (solid [50th percentile] and dotted [5th and 95th percentile] lines) are within the $95 \%$ confidence interval of the 5 th, 50th and 95th percentiles of FVIII:C response that are predicted by the final model (shaded areas), indicating absence of bias. The VPC also shows that the model does not underpredict or overpredict the variability of FVIII:C response, since $\sim 5 \%$ of measured FVIII:C responses (open circles) is less than the $95 \%$ confidence interval of the predicted 5 th percentile or more than the $95 \%$ confidence interval of the predicted 95th percentile of FVIII:C responses. FVIII, factor VIII; FVIII:C, response of factor VIII activity; VPC, visual predictive check.

\section{Population Pharmacokinetic Modelling}

The two-compartment model was superior to the one-compartment model in describing FVIII:C increase and was therefore used as the basic model. The model comprised a first-order rate constant $\left(\mathrm{k}_{\mathrm{a}}\right)$ describing the rate of the increase of FVIII:C following desmopressin administration and the apparent PK parameters V1, CL, V2 and Q (- Fig. 3). IIV could be estimated for baseline FVIII:C, V1 and CL; the respective values were $81,67 \%$ and $86 \%$. Intraindividual variability could be estimated for baseline FVIII:C and for V1, 29 and 38\%, respectively (-Table 2 ).

\section{Covariate Analysis}

For all tested covariates at least $73 \%$ of data were available (-Tables 1 and 3). However, for VWF:Ag during desmopressin administration, platelet count and aPTT less than $60 \%$ of data were available and these variables were therefore excluded from the covariate analysis. The multivariate analysis showed FVIII-recent ( $85 \%$ of data available) to be strongly positively correlated with estimated baseline FVIII:C $(p<0.001)$ and negatively correlated with V1 and CL $(p<0.001$ for both) (see - Table 3 for the univariate analysis). The identified correlations are reflected by three equations (-Table 2), which are derived from Eqs. 4 and 5 in -Supplementary Table 1 (where these equations are further explained).

-Fig. 4 illustrates the effect of these covariates on the FVIII:C response-time curve according to the final model for five typical patients with all median characteristics of the population, except for their FVIII-recent. For FVIII-recent, the 5th, 25th, 75th and 95th percentiles and the median were chosen. A higher FVIII-recent correlates with a higher baseline FVIII and with a higher absolute increase of FVIII:C after 


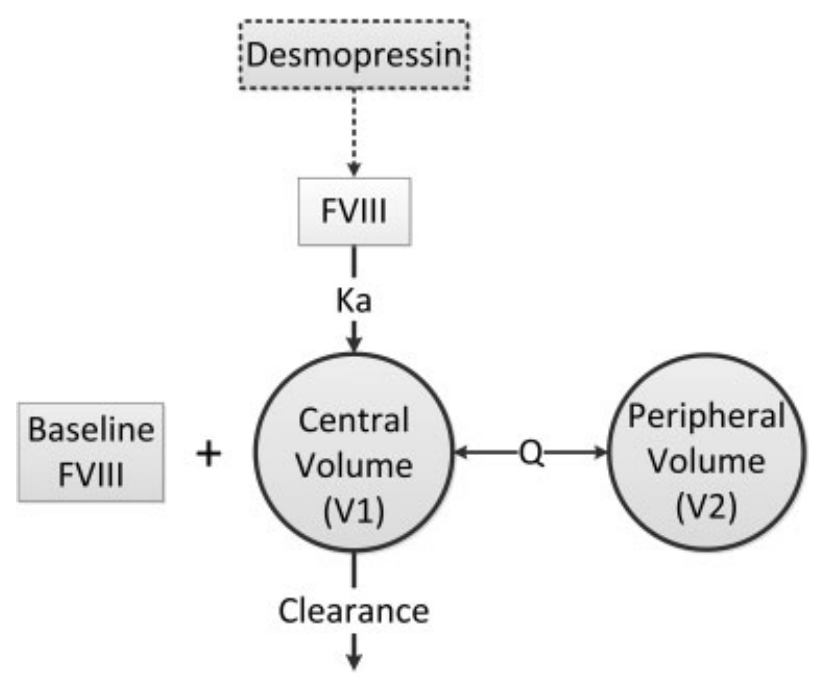

Fig. 3 Two-compartment model for FVIII after desmopressin administration. FVIII, factor VIII; $\mathrm{K}_{\mathrm{a}}$, first-order rate constant describing the rate of the FVIII level increase after desmopressin administration; estimated baseline FVIII, estimated FVIII:C directly before desmopressin administration; Q, intercompartmental clearance.

desmopressin administration. It also correlates with a longer duration of the FVIII:C response above 0.50 and $0.30 \mathrm{IU} / \mathrm{mL}$.

Upon implementation of the significant covariates in the final population model, IIV in baseline FVIII:C decreased from 81 to $37 \%$. IIV of V1 and CL decreased from 67 to $43 \%$ and 86 to $50 \%$, respectively. The model fits the data adequately, which is shown in the VPC (-Fig. 2). The data as measured (dotted and solid lines) fall within the predicted values (shaded areas), indicating absence of bias. Good-of-fit is also shown in -Supplementary Fig. 1-c.

\section{FVIII:C Response to Desmopressin}

By using the final and validated model, the defined measures of FVIII:C response to desmopressin were calculated. The median absolute increase in FVIII:C was $0.47 \mathrm{IU} / \mathrm{mL}$ (IQR: $0.32-0.65 \mathrm{IU} / \mathrm{mL}, n=142$ ). Half-life of FVIII:C had a median of 5.8 hours with an IQR of 4.2 to 7.9 hours. In 128 (90\%) desmopressin administrations, peak FVIII:C was $>0.30 \mathrm{IU} / \mathrm{mL}$. Median duration of FVIII:C $>0.30 \mathrm{IU} / \mathrm{mL}$ was 9.8 hours (IQR of 4.3-22.6 hours). A peak FVIII:C $>0.50 \mathrm{IU} / \mathrm{mL}$ was reached in $63 \%$ of patients. The FVIII:C remained above $0.50 \mathrm{IU} / \mathrm{mL}$ in these patients for a median time of 4.8 hours (IQR of 2.1-10.7 hours). FVIII:C $>0.50 \mathrm{IU} / \mathrm{mL}$ for more than 24 hours was seen in 11 patients (7.7\%); FVIII:C $>0.30 \mathrm{IU} / \mathrm{mL}$ in 29 patients (20\%).

In the 14 patients with repeated desmopressin administrations, the median time between administrations was 30 months (IQR: 17-129 months). In 6 out of 14 (43\%), a reproducible response was observed. In three (21\%) patients, a higher second peak FVIII:C was seen, whereas five (36\%) other patients had a lower peak FVIII:C. The maximum difference was $58 \%$ between administrations. All individual responses are shown in - Fig. $\mathbf{5}$.

\section{Discussion}

We have assessed the population PK of FVIII:C following desmopressin administration in 128 nonsevere HA patients. Our model shows a high IIV in FVIII:C response. In addition, our results suggest that the FVIII: $C$ response to desmopressin is not reproducible in all patients. Only 6 out of 14 of patients with repeated desmopressin administrations had similar peak FVIII:C values after both administrations.

The high IIV could partially be explained by FVIII-recent. FVIII-recent had a positive correlation with baseline FVIII:C and a negative correlation with V1 and clearance. Previous studies found FVIII-lowest and not FVIII-recent to be predictive of the FVIII:C response, although FVIII-recent was never defined as a separate covariate. ${ }^{4,6,19}$ Both FVIII-recent and FVIII-lowest were not influenced by previous treatment with FVIII concentrate or desmopressin and are the result of endogenous synthesis. Therefore, both parameters could be related to the response to desmopressin. Why FVIII-recent was found rather than FVIII-lowest may be explained by the fact that FVIII:C increases over time in patients with nonsevere $\mathrm{HA}(0.0064 \mathrm{IU} / \mathrm{mL}$ per decade $)$ and is therefore a better marker for current endogenous capacity. ${ }^{5,8,20-27}$ FVIII-recent could not explain the IIV entirely. This might mean that other covariates are of interest. One candidate may be the exposure to desmopressin as that would enable a better description and prediction of the FVIII:C response in theory. Unfortunately, desmopressin plasma levels were not available. Two previous studies investigated the relationship between desmopressin plasma levels and the FVIII: $C$ response in healthy volunteers $(N=10)$ and HA patients $(N=14)$ and found no correlation. $^{28,29}$ Both studies hypothesized their findings could be due to a ceiling effect of desmopressin. Further research is needed to investigate this.

Interestingly, we did not find significant associations for two other covariates which previously have been described to correlate with the FVIII:C response. First, we studied age extensively as a covariate, but it was not significantly correlated with any of the PK parameters. Contrary to our findings, Di Perna et al found more responders in groups with an increasingly higher age after dividing their patient population in age groups of 10 years $(N=75) .{ }^{4}$ Nolan et al reported similar findings. ${ }^{30}$ Castaman et al only found age to be a significant covariate in a subgroup of patients with a specific mutation. ${ }^{5}$ The different findings may be explained by the fact that none of these studies used PK modelling to test covariates. In addition, FVIII-recent was included as a covariate in our study and FVIII:C levels are correlated with age. $^{20}$ Therefore, it might be possible that a correlation between FVIII response and age of previous studies is explained by a change in FVIII levels over time.

Second, VWF was not a covariate in our model. VWF protects FVIII from clearance and is also released from the endothelium in response to desmopressin. ${ }^{1,25}$ Therefore, we expected VWF to have an influence on FVIII:C response. However, Castaman et al studied the influence of VWF on FVIII:C response in HA patients and actually found a negative correlation between VWF:Ag and peak FVIII:C level in 
Table 2 Parameter estimates of the final FVIII population pharmacokinetic model (with covariates) and the model without covariates

\begin{tabular}{|c|c|c|c|c|}
\hline \multirow[t]{2}{*}{ Parameter } & \multicolumn{2}{|c|}{ Model without covariates } & \multicolumn{2}{|c|}{ Final model with covariates } \\
\hline & Estimate & RSE (\%) & Estimate & RSE (\%) \\
\hline Baseline FVIII parameter (IU/mL) & 0.14 & 6.9 & 0.15 & 4.6 \\
\hline $\mathrm{k}_{\mathrm{a}}(\mathrm{h}-1)$ & 3.8 & 16 & 3.8 & 24 \\
\hline V1 (L) & 1.8 & 6.4 & 1.7 & 6.9 \\
\hline$C L(L / h)$ & 0.26 & 7.8 & 0.26 & 6.5 \\
\hline V2 (L) & 0.23 & 23 & 0.24 & 36 \\
\hline $\mathrm{Q}(\mathrm{L} / \mathrm{h})$ & 0.13 & 21 & 0.11 & 48 \\
\hline \multicolumn{5}{|l|}{ Interindividual variability ${ }^{\mathrm{a}}$} \\
\hline Baseline FVIII parameter (\%) & 81 & 16 & 37 & 99 \\
\hline V1 (\%) & 67 & 27 & 43 & 33 \\
\hline $\mathrm{CL}(\%)$ & 86 & 17 & 50 & 22 \\
\hline Correlation IIV baseline FVIII parameter and IIV V1 & -0.89 & 18 & -0.87 & 34 \\
\hline \multicolumn{5}{|l|}{ Intraindividual variability ${ }^{\mathrm{a}}$} \\
\hline Baseline FVIII parameter (\%) & 29 & 47 & 38 & 71 \\
\hline V1 (\%) & 38 & 39 & 38 & 28 \\
\hline \multicolumn{5}{|l|}{ Residual variability } \\
\hline Proportional error (\%) & 12 & 9.7 & 12 & 9.7 \\
\hline Additive error $(\mathrm{IU} / \mathrm{mL})$ & 0.018 & 19 & 0.018 & 20 \\
\hline \multicolumn{5}{|l|}{ Covariate correlations } \\
\hline FVIII ${ }_{\text {recent}}$-baseline FVIII parameter & - & - & 0.74 & 18 \\
\hline Correction factor when FVIII $I_{\text {recent }}$ is not available & - & - & 1.2 & 15 \\
\hline $\mathrm{FVIIII}_{\text {recent }}-\mathrm{V} 1$ & - & - & -0.61 & 16 \\
\hline Correction factor when FVIII $I_{\text {recent }}$ is not available & - & - & 1.1 & 15 \\
\hline FVIII ${ }_{\text {recent }}-\mathrm{CL}$ & - & - & -0.73 & 10 \\
\hline Correction factor when FVIII $I_{\text {recent }}$ is not available & - & - & 0.78 & 17 \\
\hline
\end{tabular}

Abbreviations: CL, apparent FVIII clearance; IIV, interindividual variability; FVIII, factor VIII; IIV, interindividual variability; IOV, interoccasion variability; $\mathrm{k}_{\mathrm{a}}$, first-order rate constant describing the rate of the FVIII level increase after desmopressin administration; Q, apparent intercompartmental clearance; RSE, relative standard error; V1, apparent central volume of distribution; V2, apparent peripheral volume of distribution. Note: Equations for the identified correlations are as follows:

Estimated baseline $\mathrm{FVIII}_{\mathrm{ij}}=0.15 \times(\mathrm{FVIII-recent} / 0.15)^{(0.74 \times \text { missing FVIIIrecentij) }} \times 1.2^{(1-\text { missing FVIII-recentij) }}($ Eq. 1)

FVIII CL $L_{i j}=0.26 \times(F V I I I-r e c e n t / 0.15)^{(-0.73 \times \text { missing FVIIIrecentij })} \times 0.77^{(1-\text { missing FVIIIrecentij) }}($ Eq. 2)

$F V I I I V 1_{i j}=1.7 \times\left(\right.$ FVIII-recent/0.15) ${ }^{(-0.61 \times \text { missing FVIIIrecentij) }} \times 1.1^{(1-\text { missing FVIIIrecentij) }}($ Eq. 3).

aIIV and intraindividual variability estimates are expressed as coefficients of variation.

patients with an identified $F 8$-gene mutation. ${ }^{5}$ The reasons we did not find a correlation may be the missing VWF data after desmopressin administrations and the lack of representativeness of historical VWF values.

Median half-life of FVIII after desmopressin administration in this cohort (median 5.8 hours) was shorter than halflife from FVIII concentrates reported in literature $(\sim 8-$ 12 hours). In a previous study, half-life of FVIII:C after desmopressin infusion ranged from 1.3 to 11.8 hours in a subset of HA patients. ${ }^{5}$ Ludlam et al found a FVIII half-life after desmopressin of approximately 4.5 hours in healthy subjects and 3 hours in HA patients. ${ }^{31}$ Difference in half-life between FVIII concentrate and endogenous FVIII after desmopressin may be due to the mutation in endogenous FVIII, especially as half-life after desmopressin seems lower in patients than in healthy subjects. However, Mannucci et al showed the opposite as they found a half-life of 4.4 hours in healthy subjects and 11.2 hours in HA patients. ${ }^{32}$ This suggests that other mechanisms may play a role, but more research is needed is to investigate this.

Another difference with previous research is that we found a reproducibility in response of $43 \%$, which was $15 \%$ lower than reported previously. Rodeghiero et al found a reproducibility of $70 \%$ among $10 \mathrm{HA}$ patients. ${ }^{9}$ However, they defined reproducibility as a deviation of peak FVIII:C on one occasion relative to the average peak FVIII:C in that patient, with a margin of $20 \%$. In contrast, we used a difference between peak FVIII:C of maximally $25 \%$, as this value is 
Table 3 Tested covariates in univariate analysis

\begin{tabular}{|c|c|c|c|}
\hline \multirow[t]{2}{*}{ Covariate } & \multicolumn{3}{|c|}{ Outcome univariate analysis } \\
\hline & Baseline FVIII & $\mathrm{CL}$ & V1 \\
\hline \multicolumn{4}{|c|}{ Mutation domain } \\
\hline A1 & NS & NS & NS \\
\hline $\mathrm{A} 2$ & NS & NS & NS \\
\hline A3 & NS & $p<0.05$ & NS \\
\hline B & NS & NS & NS \\
\hline C1 & $p<0.001$ & $p<0.01$ & NS \\
\hline $\mathrm{C} 2$ & NS & NS & NS \\
\hline FVIII-recent & $p<0.001$ & $p<0.001$ & $p<0.01$ \\
\hline
\end{tabular}

Abbreviations: CL, apparent FVIII clearance; FVIII-recent, recently measured FVIII:C; NS, nonsignificant; V1, apparent central volume of distribution.

Note: Age, weight, administration mode, blood type, FVIII-lowest, most recently measured von Willebrand factor antigen, desmopressin dose and indication for desmopressin (test, bleeding and prophylaxis before surgery) were no significant covariates. aPTT, platelet count and von Willebrand factor antigen during desmopressin administration were not tested as covariates due to too many ( $>40 \%)$ missing data.

used in the European bioequivalence guideline. ${ }^{18}$ If this definition is applied to the data of Rodeghiero et al, $60 \%$ of patients would have had a reproducible FVIII:C response. In addition, the median time between desmopressin was higher

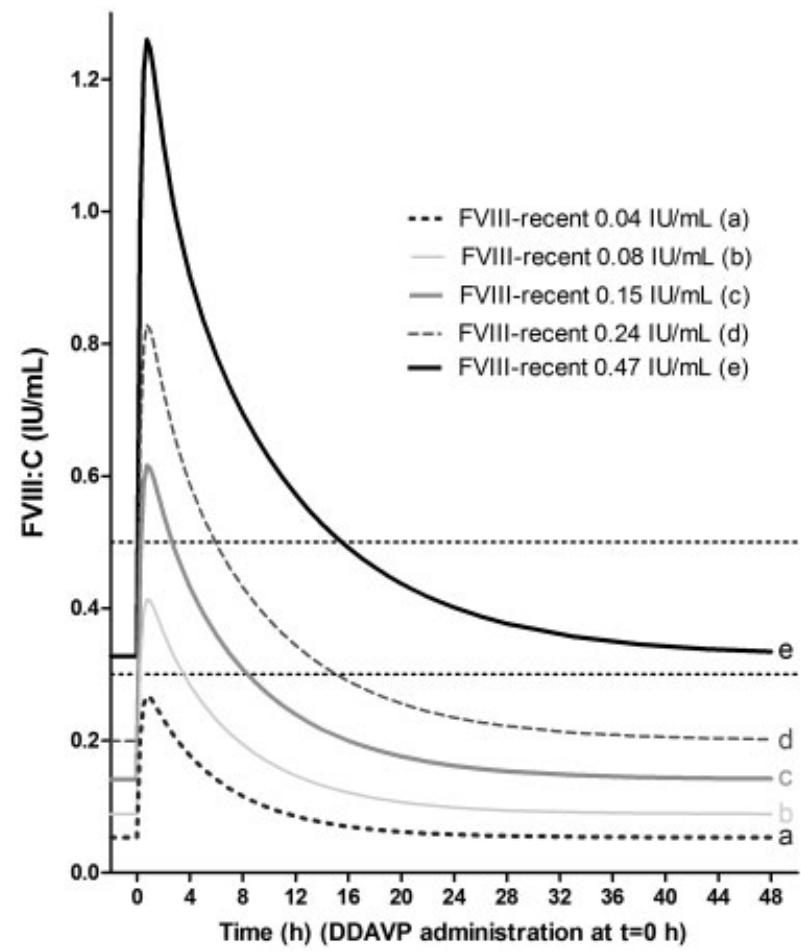

Fig. 4 FVIII:C response-time curves according to the final model. Response-time curves for five typical patients with all median characteristics of the population, but with a FVIII-recent of 0.04 (5th percentile), 0.08 (25th percentile), 0.15 (median), $0.24 \mathrm{IU} / \mathrm{mL}$ (75th percentile) or 0.47 (95th percentile). DDAVP, desmopressin; FVIII, factor VIII; FVIII:C, response of factor VIII activity.

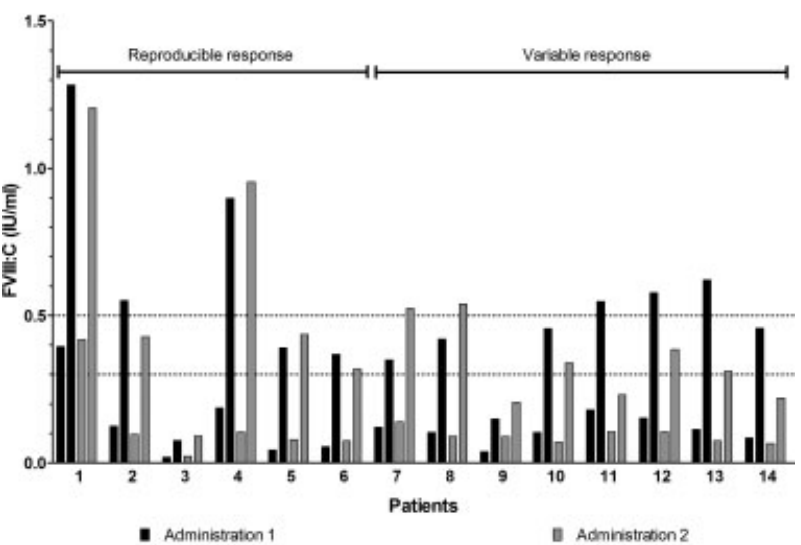

Fig. 5 Reproducibility of FVIII:C to desmopressin. Individual response showing baseline FVIII:C (left bar) and peak FVIII:C (right bar) for both the first (black) and second (grey) desmopressin administration. FVIII:C, response of factor VIII activity.

in our population (30 months) than in the previous study (14 months). ${ }^{9}$ This may indicate that response to desmopressin changes over a longer period of time.

Our findings may have treatment implications. Currently, FVIII: $C$ response is often not monitored in patients with an adequate previous response to desmopressin. This might be reconsidered as FVIII-recent seems more predictive for response than FVIII-lowest and as 5 out of 14 patients had a significantly lower response after a second desmopressin administration at a different occasion.

Regarding the observed reproducibility in our cohort, it seems that patients with a low response were more likely to be re-evaluated, as FVIII-recent and FVIII:C response were lower in patients with two desmopressin administrations than in patients with one desmopressin administration (data not shown). Given this aspect and the fact that reproducibility could only be assessed in 14 patients, our findings may not represent the reproducibility of testing in the entire cohort. Unfortunately, we were not able to identify covariates that may predict which patients are at risk of a nonreproducible second response, most likely as a result of lack of statistical power due to the small sample size.

Nevertheless, the results on reproducibility might suggest that measuring a trough and peak FVIII:C level around (surgical) interventions may be considered in some patients, also when results of earlier FVIII:C response after desmopressin administration are available, depending on the consequences of bleeding during or after a specific intervention. More specific recommendations can only be made when our results are confirmed in a larger dataset, as also the only other study looking into reproducibility included a low number of patients. ${ }^{9,10}$

A few other limitations were present in this study. First, data collection was performed retrospectively. Therefore, not all parameters were available in each patient, leading to missing data. This may have limited the power for the identification of associations between PK parameters and covariates, for example, VWF. Second, F8-gene mutations 
were divided into six groups according to their domains: A1, $\mathrm{A} 2, \mathrm{~B}, \mathrm{~A} 3, \mathrm{C} 1$ and $\mathrm{C} 2$. We did not study individual mutations as the subgroups would become too small. Therefore, the influence of some mutations may have been missed, as different mutations in the same domain may have opposite effects. Third, our model is an empirical model describing only the data. A mechanistic model might give more insight into the physiology of FVIII after desmopressin administration. A final limitation could have been that all data were analysed without dividing the population into groups according to the indication for desmopressin administration (test, bleeding and prophylaxis). However, as treatment indication was not a significant covariate, separate analysis would not yield different results, while statistical power would be diminished.

Overall, an adequate population PK model was constructed that describes desmopressin response in nonsevere HA patients. Much IIV remains unexplained, despite identification of the most recently FVIII:C as significant covariate. In addition, reproducibility of response may be lower than previously thought. Therefore, a desmopressin test dose in each patient remains necessary. In addition, monitoring of FVIII:C response to desmopressin around (surgical) interventions or in case of bleeding may be considered in some patients. More research is needed to investigate the reproducibility of response to desmopressin.

\section{What is known about this topic?}

- The FVIII:C response to desmopressin varies between HA patients.

- The FVIII:C response to desmopressin remains difficult to predict.

\section{What does this paper add?}

- PK modelling is useful to describe the FVIII:C response to desmopressin in haemophilia A.

- The most recent FVIII:C level influences the FVIII:C response.

- The FVIII:C response may not be reproducible in some HA patients.

\section{Conflict of Interest}

L.M. Schütte received reimbursement from CSL-Behring for attending a symposium.

R.M. van Hest and S.C.M. Stoof have no conflict of interest. F.W.G. Leebeek received unrestricted research grants from CSL-Behring and Baxalta/Shire not related to this study.

M.H. Cnossen received unrestricted research/educational funding for various projects as well as travel fees from the following institutions and companies: ZonMW, Innovatiefonds, Pfizer, Baxalta/Shire, Bayer Schering Pharma, Novo Nordisk, Novartis and CSL Behring, all not related to this study.
M.J.H.A. Kruip received unrestricted research grants from Pfizer, Innovatiefonds, Ferring and ZonMW with no involvement in this study.

R.A.A. Mathôt received personal fees from Merck Sharp \& Dohme and Zeria and grants from Bayer, UCB Pharma and Hoffman La Roche with no involvement in this study.

\section{Authors' Contributions}

M.J.H.A. Kruip and R.A.A. Mathot designed the study, interpreted data, and critically revised the article. L.M. Schütte performed research, analysed and interpreted the data, and wrote the article. R.M. van Hest analysed and interpreted the data, and wrote the article. S.C.M. Stoof performed research, interpreted data, and critically revised the article. F.W.G. Leebeek and M.H. Cnossen interpreted data and critically revised the article.

\section{References}

1 Kaufmann JE, Vischer UM. Cellular mechanisms of the hemostatic effects of desmopressin (DDAVP). J Thromb Haemost 2003;1(04): 682-689

2 Turner NA, Moake JL. Factor VIII is synthesized in human endothelial cells, packaged in Weibel-Palade bodies and secreted bound to ULVWF strings. PLoS One 2015;10(10):e0140740

3 Stoof SC, Cnossen MH, de Maat MP, Leebeek FW, Kruip MJ. Side effects of desmopressin in patients with bleeding disorders. Haemophilia 2016;22(01):39-45

4 Di Perna C, Riccardi F, Franchini M, Rivolta GF, Pattacini C, Tagliaferri A. Clinical efficacy and determinants of response to treatment with desmopressin in mild hemophilia a. Semin Thromb Hemost 2013;39(07):732-739

5 Castaman G, Mancuso ME, Giacomelli SH, et al. Molecular and phenotypic determinants of the response to desmopressin in adult patients with mild hemophilia A. J Thromb Haemost 2009;7(11):1824-1831

6 Lethagen S. Desmopressin in mild hemophilia A: indications, limitations, efficacy, and safety. Semin Thromb Hemost 2003;29 (01):101-106

7 Stoof SC, Sanders YV, Petrij F, et al. Response to desmopressin is strongly dependent on F8 gene mutation type in mild and moderate haemophilia A. Thromb Haemost 2013;109(03): 440-449

8 Nance D, Fletcher SN, Bolgiano DC, Thompson AR, Josephson NC, Konkle BA. Factor VIII mutation and desmopressin-responsiveness in 62 patients with mild haemophilia A. Haemophilia 2013; 19(05):720-726

9 Rodeghiero F, Castaman G, Di Bona E, Ruggeri M. Consistency of responses to repeated DDAVP infusions in patients with von Willebrand's disease and hemophilia A. Blood 1989;74(06): 1997-2000

10 Björkman S. Limited blood sampling for pharmacokinetic dose tailoring of FVIII in the prophylactic treatment of haemophilia A. Haemophilia 2010;16(04):597-605

11 Carlsson M, Berntorp E, Björkman S, Lindvall K. Pharmacokinetic dosing in prophylactic treatment of hemophilia A. Eur J Haematol 1993;51(04):247-252

12 Hazendonk H, Fijnvandraat K, Lock J, et al; "OPTI-CLOT" study group. A population pharmacokinetic model for perioperative dosing of factor VIII in hemophilia A patients. Haematologica 2016;101(10):1159-1169 
13 Ette EI, Ludden TM. Population pharmacokinetic modeling: the importance of informative graphics. Pharm Res 1995;12(12): 1845-1855

14 Keizer RJ, Karlsson MO, Hooker A. Modeling and simulation workbench for NONMEM: Tutorial on Pirana, PsN, and Xpose. CPT Pharmacometrics Syst Pharmacol 2013;2:e50

15 Jonsson EN, Karlsson MO. Xpose-an S-PLUS based population pharmacokinetic/pharmacodynamic model building aid for NONMEM. Comput Methods Programs Biomed 1999;58(01): 51-64

16 Lindbom L, Pihlgren P, Jonsson ENiclas. PsN-Toolkit-a collection of computer intensive statistical methods for non-linear mixed effect modeling using NONMEM. Comput Methods Programs Biomed 2005;80(03):277

17 World Federation of Hemophilia. Plasma factor level and duration of administration. Available at: http://www1.wfh.org/publications/files/pdf-1500.pdf. Accessed October 9, 2015

18 Morais JA, Lobato MdoR. The new European Medicines Agency guideline on the investigation of bioequivalence. Basic Clin Pharmacol Toxicol 2010;106(03):221-225

19 Leissinger C, Carcao M, Gill JC, Journeycake J, Singleton T, Valentino L. Desmopressin (DDAVP) in the management of patients with congenital bleeding disorders. Haemophilia 2014;20(02): 158-167

20 Loomans JI, van Velzen AS, Eckhardt CL, et al. Variation in baseline factor VIII concentration in a retrospective cohort of mild/moderate hemophilia A patients carrying identical F8 mutations. J Thromb Haemost 2017;15(02):246-254

21 Hsu TC, Pratt KP, Thompson AR. The factor VIII C1 domain contributes to platelet binding. Blood 2008;111(01):200-208

22 Lü J, Pipe SW, Miao H, Jacquemin M, Gilbert GE. A membraneinteractive surface on the factor VIII C1 domain cooperates with the C2 domain for cofactor function. Blood 2011;117(11): 3181-3189

23 Chiu PL, Bou-Assaf GM, Chhabra ES, et al. Mapping the interaction between factor VIII and von Willebrand factor by electron microscopy and mass spectrometry. Blood 2015;126(08): 935-938

24 Ebberink EH, Bouwens EA, Bloem E, et al. Factor VIII/V C-domain swaps reveal discrete C-domain roles in factor VIII function and intracellular trafficking. Haematologica 2017;102(04):686-694

25 Lenting PJ, VAN Schooten CJ, Denis CV. Clearance mechanisms of von Willebrand factor and factor VIII. J Thromb Haemost 2007;5 (07):1353-1360

26 Lenting PJ, van Mourik JA, Mertens K. The life cycle of coagulation factor VIII in view of its structure and function. Blood 1998;92 (11):3983-3996

27 Seary ME, Feldman D, Carcao MD. DDAVP responsiveness in children with mild or moderate haemophilia A correlates with age, endogenous FVIII:C level and with haemophilic genotype. Haemophilia 2012;18(01):50-55

28 Lethagen S, Harris AS, Sjörin E, Nilsson IM. Intranasal and intravenous administration of desmopressin: effect on F VIII/vWF, pharmacokinetics and reproducibility. Thromb Haemost 1987;58 (04):1033-1036

29 Mannucci PM, Vicente V, Alberca I, et al. Intravenous and subcutaneous administration of desmopressin (DDAVP) to hemophiliacs: pharmacokinetics and factor VIII responses. Thromb Haemost 1987;58(04):1037-1039

30 Nolan B, White B, Smith J, O'Reily C, Fitzpatrick B, Smith OP. Desmopressin: therapeutic limitations in children and adults with inherited coagulation disorders. Br J Haematol 2000;109 (04):865-869

31 Ludlam CA, Peake IR, Allen N, Davies BL, Furlong RA, Bloom AL. Factor VII and fibrinolytic response to deamino-8-D-argenine vasopressin in normal subjects and dissociate response in some patients with haemophilia and von Willebrand's disease. Br J Haematol 1980;45(03):499-511

32 Mannucci PM, Canciani MT, Rota L, Donovan BS. Response of factor VIII/von Willebrand factor to DDAVP in healthy subjects and patients with haemophilia A and von Willebrand's disease. $\mathrm{Br}$ J Haematol 1981;47(02):283-293 